\section{Brain ultrasonographic findings of late-onset circulatory dysfunction due to adrenal insufficiency in preterm infants}

\author{
Su-Mi Shin, Jee Won Chai \\ Department of Radiology, SMG-SNU Boramae Medical Center, Seoul National University \\ College of Medicine, Seoul, Korea
}

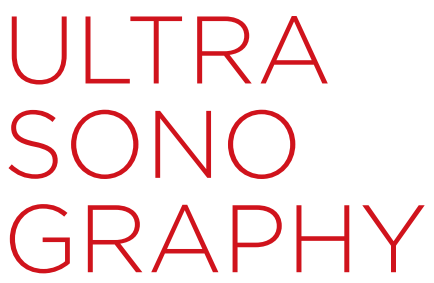

ORIGINAL ARTICLE

http://dx.doi.org/10.14366/usg.16013 pISSN: 2288-5919 - eISSN: 2288-5943 Ultrasonography 2016;35:258-264

Received: March 6, 2016

Revised: April 14, 2016

Accepted: April 18, 2016

Correspondence to: Jee Won Chai, MD, Department of Radiology, SMG-SNU Boramae Medical Center, Seoul National University College of Medicine, 20 Boramae-ro 5-gil, Dongjak-gu, Seoul 07061, Korea

Tel. $+82-2-870-2549$

Fax. +82-2-870-3539

E-mail: chaijw@gmail.com

This is an Open Access article distributed under the terms of the Creative Commons Attribution NonCommercial License (http://creativecommons.org/ licenses/by-nc/3.0/) which permits unrestricted noncommercial use, distribution, and reproduction in any medium, provided the original work is properly cited.

Copyright (C) 2016 Korean Society of Ultrasound in Medicine (KSUM)

\section{Introduction}

Late-onset systemic hypotension and oliguria without obvious cause following stable circulation and respiration are not rare in preterm infants, and such episodes are referred to as late-onset circulatory dysfunction (LCD) of premature infants [1]. LCD due to adrenal insufficiency (AI) is one of the major causes of late-onset hypotension [2]. Recently, several studies performed in Japan have reported that preterm infants develop LCD that responds to glucocorticoid therapy, but not to volume expansion or vasopressors, after the first week of life [3-6]. The main problem associated with LCD is the subsequent onset of periventricular leukomalacia (PVL) and poorer neurologic development $[1,3]$. The

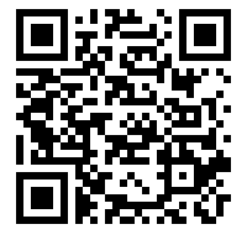

Shin SM, Chai JW. Brain ultrasonographic findings of late-onset circulatory dysfunction due to adrenal insufficiency in preterm infants. Ultrasonography. 2016 Jul;35(3):258-264.
How to cite this article: 
purpose of this study was to characterize the brain ultrasonographic findings of $L C D$ due to $A l$ in preterm infants.

\section{Materials and Methods}

This retrospective study was performed with the approval and under the guidelines of the Institutional Review Board. Informed consent was waived.

\section{Definition of $L C D$}

The definition of $L C D$ due to $A l$ is the acute onset of hypotension or oliguria requiring treatment without obvious causes such as sepsis, patent ductus arteriosus (PDA), or intraventricular hemorrhage (IVH) in preterm infants with circulatory and respiratory conditions that had been stable for several days. Such sudden collapse was resistant to conventional treatment using volume expanders and inotropic agents, whereas there was a rapid response to intravenous steroids (glucocorticoids) $[1,3]$. We defined late-onset hypotension as a decrease in systolic blood pressure to below $30 \mathrm{~mm} \mathrm{Hg}$ occurring after the first week of life, and oliguria as a decrease in the urine output to $\leq 0.5 \mathrm{~mL} / \mathrm{kg} / \mathrm{hr}$ over the past 8 hours or to $\leq 1.0 \mathrm{~mL} / \mathrm{kg} / \mathrm{hr}$ over the past 24 hours $[2,7]$.

\section{Patients}

Between December 2009 and February 2014, 257 preterm infants with gestational age (GA) less than 33 weeks were hospitalized in our neonatal intensive care unit (NICU). Thirty-five preterm infants were diagnosed with Al based on clinical and laboratorial findings. Among them, 21 infants were excluded for the following reasons: death ( $n=2)$, early AI ( $n=5,<1$ week), sepsis $(n=1)$, and PDA ( $n=13)$. Among the cases of PDA, a state of closure after using indomethacin $(n=8)$ or a ligation operation $(n=3)$, and sustained PDA $(n=2)$ were all excluded. The ultimate study group comprised 14 infants.

Fourteen of 257 infants (5.4\%) were diagnosed with LCD due to Al. They were six male and eight female infants and nine infants $(64 \%)$ were a twin $(n=7)$ or triplet $(n=2)$. The mean $G A$ at birth was 30.6 weeks (range, 29.1 to 32.4 weeks) and the mean birth weight was 1,433 g (range, 540 to 1,790 g). The median Apgar scores at 1 minute and 5 minutes were 6.0 and 7.5 , respectively. None of the cases showed any evidence of hypoxic ischemic encephalopathy, sepsis, PDA, or IVH before the occurrence of LCD. However, five infants who experienced spontaneous closure of PDA at less than 1 week of postnatal age, before LCD, were included in this study. None of the patients had any other abnormalities on cranial ultrasonography, anomalies elsewhere in the body, or underlying disease, except two patients with mild bronchopulmonary dysplasia.

We reviewed clinical data at the date of the occurrence of LCD.

\section{Ultrasonography Image Analysis}

The ultrasonography examination was performed by one pediatric radiologist (with 10 years of experience) using an iU22 (Philips, Bothell, WA, USA) with a 5-8 $\mathrm{MHz}$ sector and 5-12 MHz linear transducers. The patients were laid supine on the bed in the NICU and not sedated. The ultrasonographic findings were retrospectively reviewed by two radiologists (with 10 and 8 years of experience, respectively) at a picture archiving and communication system workstation (Maroview, Marotech, Seoul, Korea). We reviewed the interval between the ultrasonographic examinations before and after LCD occurred, the interval between LCD and the first ultrasonography after LCD occurred, and the interval between the first ultrasonography and the next follow-up ultrasonography after LCD occurred.

In this retrospective study, the time schedule of routine brain ultrasonography in preterm infants in our institution was as follows: All preterm neonates born at $<33$ weeks of gestation routinely underwent brain ultrasonography within the first week of life and then follow-up brain ultrasonography was performed at least once a week until about 2-4 weeks after birth. In stable preterm infants after 2-4 weeks of age, the interval of follow-up ultrasonography was usually lengthened to 2-4 weeks. Serial brain ultrasonographic findings were analyzed before and after the occurrence of $L C D$, focusing on the change in periventricular echogenicity (PVE), the occurrence and date of hemorrhage, and the development of cystic changes in the white matter (cystic PVL). The definition of ultrasonography before the occurrence of LCD was the last ultrasonography before LCD.

\section{Results}

The ultrasonographic findings of 14 preterm infants with $L C D$ due to Al are shown in Table 1. LCD occurred at a median of postnatal age 18.5 days (range, 9 to 32 days) and a mean of 33.4 weeks (range, 31.9 to 35.0 weeks) of postmenstrual age. The serum cortisol concentration before hydrocortisone administration was median $5.3 \pm 2.6 \mu \mathrm{g} / \mathrm{dL}$ (range, 1.7 to $9.2 \mu \mathrm{g} / \mathrm{dL}$; normal reference, 5 to 25 $\mu \mathrm{g} / \mathrm{dL}$ ). All 14 patients were stable before the onset of $L C D$ and there was no history of birth asphyxia or hypoxic ischemic encephalopathy. The interval of ultrasonographic examination before and after LCD was a mean of 10.1 days (range, 4 to 18 days), the interval between LCD and ultrasonography was a mean of 2.6 days (range, 0 to 11 days), and the interval of next follow-up ultrasonography after LCD was a mean of 10.3 days (range, 3 to 25 days).

The last brain ultrasonographic findings before the occurrence of LCD showed grade 1 PVE in all 14 patients and germinal matrix hemorrhage $(G M H)$ with focal cystic change in one patient. Brain 
Table 1. Ultrasonographic findings of 14 preterm infants with $L C D$ due to $\mathrm{Al}$

\begin{tabular}{|c|c|c|c|c|c|c|c|c|c|}
\hline \multirow{3}{*}{ No. } & \multirow{3}{*}{ Sex } & \multirow{3}{*}{$\mathrm{GA}(w k)$} & \multirow{3}{*}{$\mathrm{BW}(\mathrm{g})$} & \multirow{3}{*}{$\begin{array}{l}\text { Age at } \\
\text { LCD } \\
\text { (day) }\end{array}$} & \multicolumn{5}{|c|}{ Serial ultrasonographic findings before and after the event of LCD } \\
\hline & & & & & \multicolumn{2}{|c|}{ Before LCD } & \multicolumn{3}{|c|}{ After LCD occurred } \\
\hline & & & & & PVE & $\mathrm{GMH}$ & PVE & GMH (day) & PVL (date) \\
\hline 1 & M & 30.3 & 1,750 & 19 & Gr 1 & - & Gr 1 & - & - \\
\hline 2 & M & 32.1 & 1,750 & 14 & Gr 1 & - & Gr 1 & - & - \\
\hline 3 & $\mathrm{~F}$ & 30.9 & 1,230 & 24 & Gr 1 & - & Gr 2 & New GMH (35) & Cystic PVL with macrocystic encephalomalacia (42nd day) \\
\hline 4 & F & 30.0 & 540 & 32 & Gr 1 & - & Gr 1 & New GMH (34) & - \\
\hline 5 & F & 29.7 & 1,350 & 18 & Gr 1 & - & Gr 1 & - & - \\
\hline 6 & F & 30.0 & 1,490 & 13 & Gr 1 & - & Gr 1 & - & - \\
\hline 7 & F & 29.1 & 1,160 & 28 & Gr 1 & - & Gr 1 & - & - \\
\hline 8 & M & 29.1 & 1,255 & 31 & Gr 1 & - & Gr 3 & New GMH (42) & Cystic PVL with macrocystic encephalomalacia (42nd day) \\
\hline 9 & $\mathrm{~F}$ & 32.4 & 1,790 & 17 & Gr 1 & - & Gr 1 & - & - \\
\hline 10 & $\mathrm{~F}$ & 31.3 & 1,640 & 15 & Gr 1 & - & Gr 2 & - & Non-cavitary PVL on MRI at 20 months (corrected age) \\
\hline 11 & $\mathrm{~F}$ & 30.6 & 1,670 & 19 & Gr 1 & - & Gr 1 & - & - \\
\hline 12 & M & 30.6 & 1,360 & 9 & Gr 1 & $\begin{array}{l}\text { GMH with } \\
\text { cystic change }\end{array}$ & Gr 1 & $\begin{array}{l}\text { Increased size } \\
\text { of GMH (16) }\end{array}$ & - \\
\hline 13 & M & 32.4 & 1,620 & 18 & Gr 1 & - & Gr 1 & - & - \\
\hline 14 & M & 29.9 & 1,450 & 19 & Gr 1 & - & Gr 1 & New GMH (19) & - \\
\hline
\end{tabular}

LCD, late-onset circulatory dysfunction; Al, adrenal insufficiency; GA, gestational age; BW, birth weight; PVE, periventricular echogenicity; GMH, germinal matrix hemorrhage; PVL, periventricular leukomalacia; Gr, grade.

ultrasonographic findings after LCD demonstrated no significant change in grade 1 PVE and no new lesions in eight (57\%), grade 1 PVE with newly appearing GMH in three (21\%) (Fig. 1C, D), and increased PVE in three (21\%) infants (Figs. 2C, D, 3E, F). Five infants $(36 \%)$ showed new development $(n=4)$ or increased size $(n=1)$ of GMH (Figs. 1C, D, 3D). The date of occurrence of GMH in the five patients was a median of 34 days (range, 16 to 42 days) after birth (Figs. 1C, D, 3D). Two of three infants with increased PVE developed cystic PVL and showed rapid progression to macrocystic encephalomalacia (Figs. 2, 3), especially in both parieto-occipital lobes. Periventricular cyst formations were noted at the 11th (Fig. 3, case 8 ) and the 18th (Fig. 2, case 3 ) days after LCD occurrence, that is, in both cases, the 42 nd day of life. The remaining one infant (case 10) with increased PVE developed non-cavitary PVL on magnetic resonance imaging (MRI) at a corrected age of 20 months.

\section{Discussion}

In this study, the incidence of LCD due to Al was $5.4 \%$. Kusuda et al. [8] reported an incidence of $L C D$ due to $\mathrm{Al}$ of $4 \%$ in 2,145 infants with very low birth weight $(\leq 1,500 \mathrm{~g})$ in Japan during a stay in the $\mathrm{NICU}$ in 2003. Choi et al. [7] reported the incidence was $7.6 \%$ in 128 preterm infants in Korea from January 2007 to August 2009.

Characteristic ultrasonographic findings in our study are the late development of GMH after 2 weeks of age, increased PVE after the occurrence of LCD, and late development of PVL. GMH-IVH in premature infants is typically diagnosed during the first days of life, $50 \%$ on the first day and $90 \%$ within the first 4 days [9]. New development or increased size of GMH in five patients occurred at 16-42 days of life in our study. Delayed hemorrhage (GMH-IVH) after 2 weeks of life may be related to postnatal events or insults and should not be overlooked.

The three patients who showed increased PVE after the occurrence of LCD all developed PVL (cystic PVL with macrocystic encephalomalacia [ $n=2]$ and non-cavitary PVL $[n=1]$ ) associated with adverse neurodevelopmental outcomes. Severe cystic PVL and rapid progression to macrocystic encephalomalacia were found unexpectedly on routine follow-up ultrasonography in two patients, although they had a good clinical response to steroid and had no other causes of hypotension except the occurrence of Al. The cystic changes were more rapid and extensive than typical PVL and predominantly involved both parieto-occipital lobes. The periventricular cyst formations were noted at the 11 th and the 18th day after $L C D$ occurred, that is, the 42 nd day of life in both of these two infants. Kobayashi et al. [1,3] reported that in infants with PVL, those with LCD had more severe MRI findings and developed more severe cerebral palsy than those without LCD. The pathogenesis of PVL development in patients with LCD is thought to be remarkable hypotension that might lead to a striking decrease in cerebral blood flow (CBF) to the white matter. They presumed the hypotension 
during LCD is associated with the development of PVL. Young et al. [10] demonstrated that the regional CBF to periventricular and occipital (visual radiations) white matter significantly decreases during severe hypotension in newborn dogs, whereas regional CBF to all gray matter structures is preserved. Although our two cases with severe cystic PVL and macrocystic encephalomalacia are well correlated with previous reports $[1,3,10,11]$, the number of patients is too small to draw conclusions.

In stable preterm infants after 2-4 weeks of age, the interval for follow-up ultrasonography was usually lengthened to 2-4 weeks. However, when LCD occurs due to $A \mathrm{I}$, the interval of ultrasonography should be shortened. Kobayashi et al. [3] reported that periventricular cysts were initially detected far later in cases of PVL with LCD than in those without LCD. This might be attributed to the decreased frequency of ultrasonography performed once an infant has reached 1 month of age. In our study, the interval of ultrasonographic examination before and after LCD was as long as 18 days (mean, 10.1 days; range, 4 to 18 days) and the interval of next follow-up ultrasonography after LCD was up to 25 days (mean, 10.3 days; range, 3 to 25 days). In seven cases, even if ultrasonography was performed shortly after LCD (the interval between $L C D$ and ultrasonography was $0-1$ day), the ultrasonographic changes were subsequently detected later because the interval of next follow-up ultrasonography after the occurrence of LCD was up to 25 days (mean, 10.3 days; range, 3 to 25 days). Therefore, if hypotension is suspected to be associated with LCD due to $\mathrm{Al}$, we think the interval of ultrasonographic examination should be shortened to 1 week, at least for 1 month after LCD occurs.

This study had several limitations, such as its retrospective design and small number of patients. Further research is needed to specify the ultrasonographic findings of LCD due to $\mathrm{Al}$ in a large population with a control group.

In conclusion, LCD due to Al may be associated with the late development of GMH (36\%) after 2 weeks of age, increased PVE (21\%) after LCD, and the development of cystic PVL with rapid progression to macrocystic encephalomalacia (14\%).

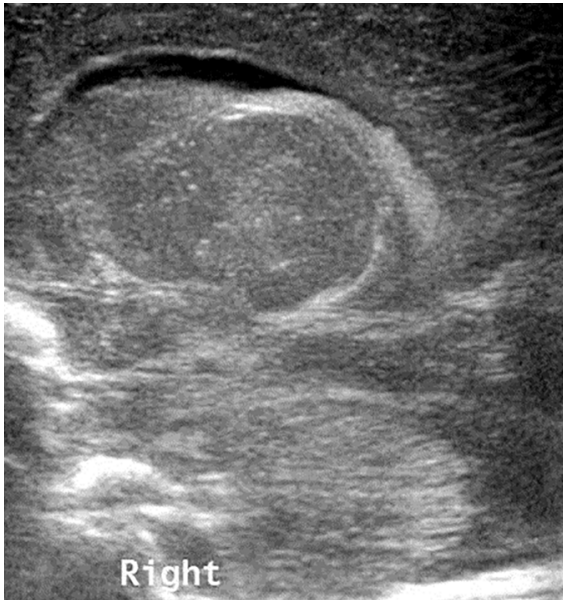

A

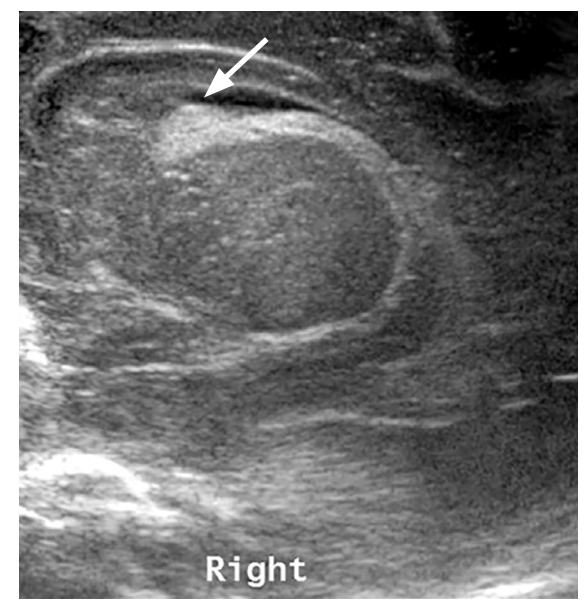

C

e-ultrasonography.org

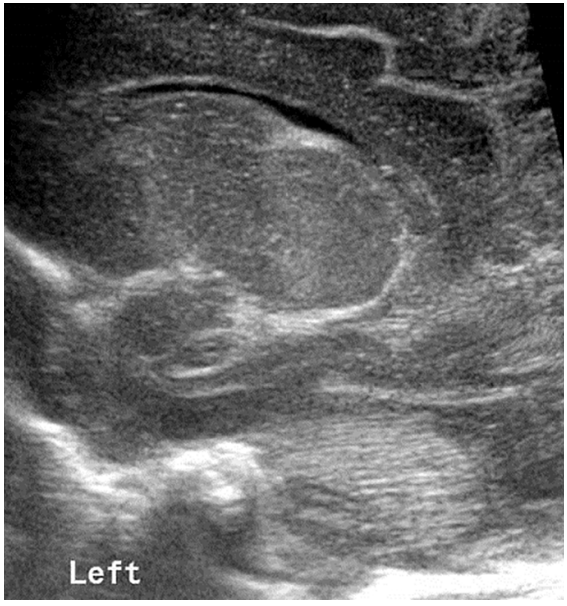

B

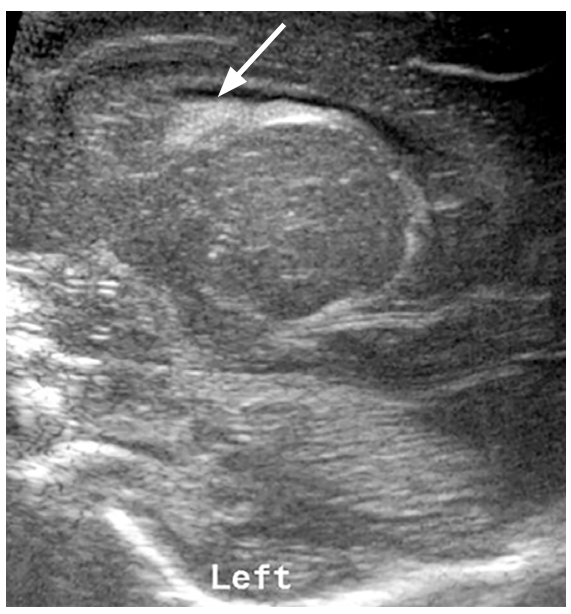

D
Fig. 1. A preterm infant (30 weeks' gestational age) with bilateral germinal matrix hemorrhage (GMH) after the occurrence of late-onset circulatory dysfunction (LCD). A, B. At age 20 days, sagittal brain sonograms show no evidence of GMH before LCD. C, D. At age 34 days, sagittal brain sonograms show newly noted bilateral GMH (arrows) after LCD which occurred at 32 days of age. 


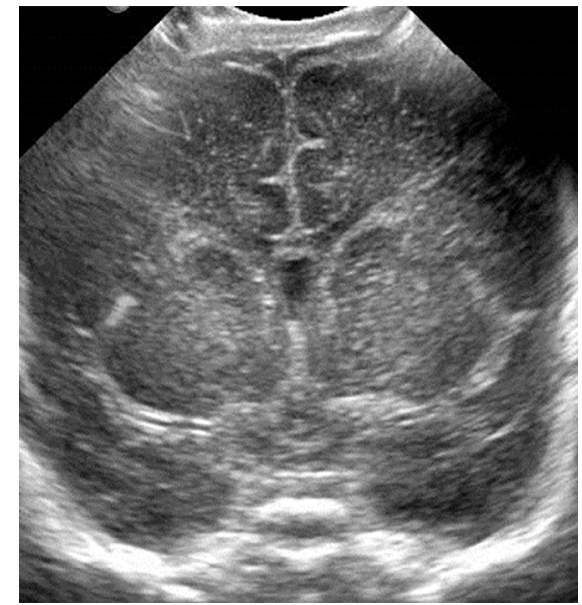

A

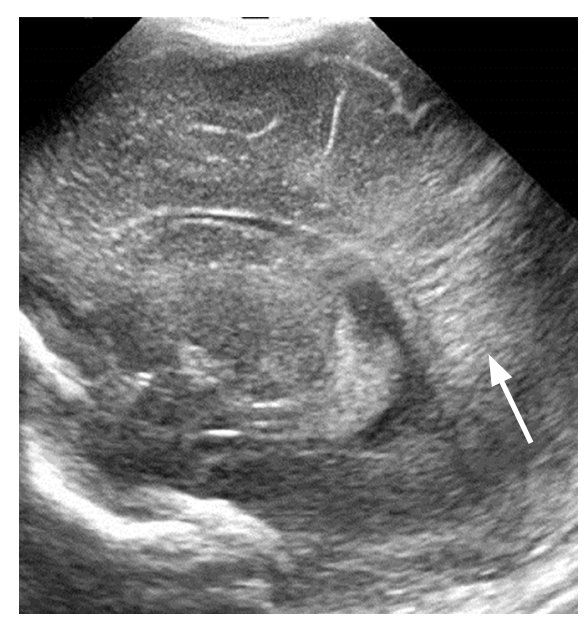

D

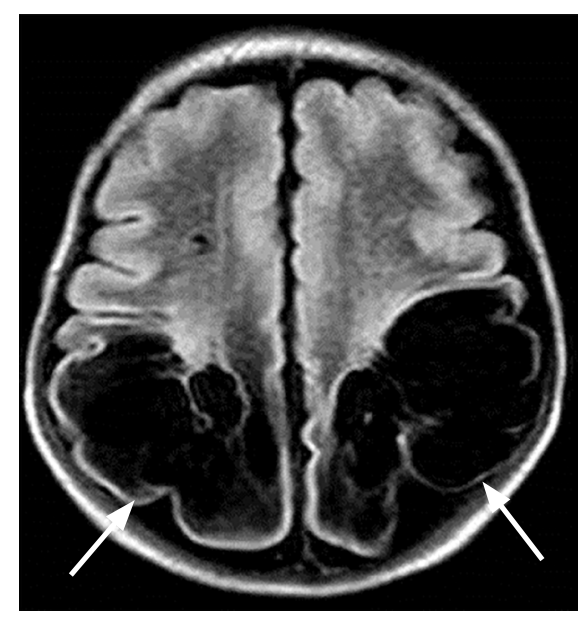

G

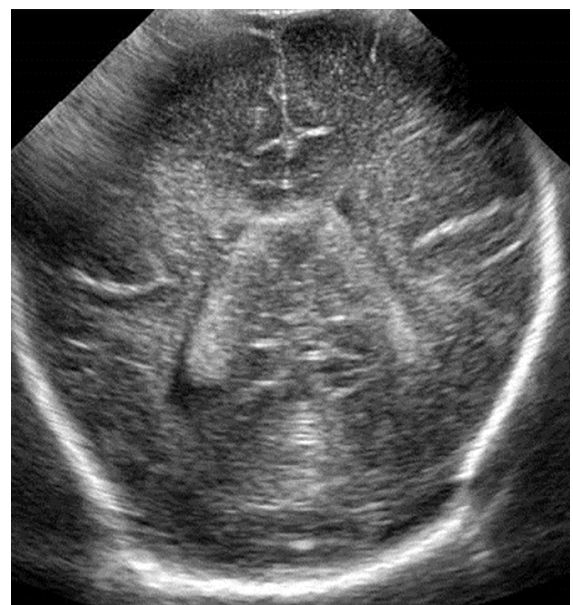

B

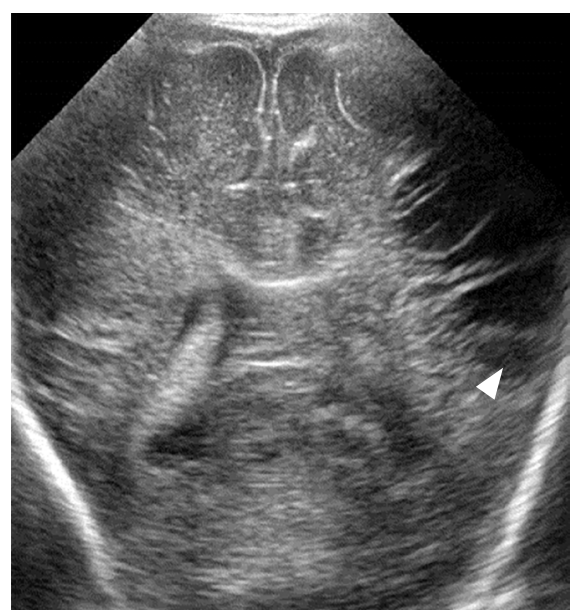

E

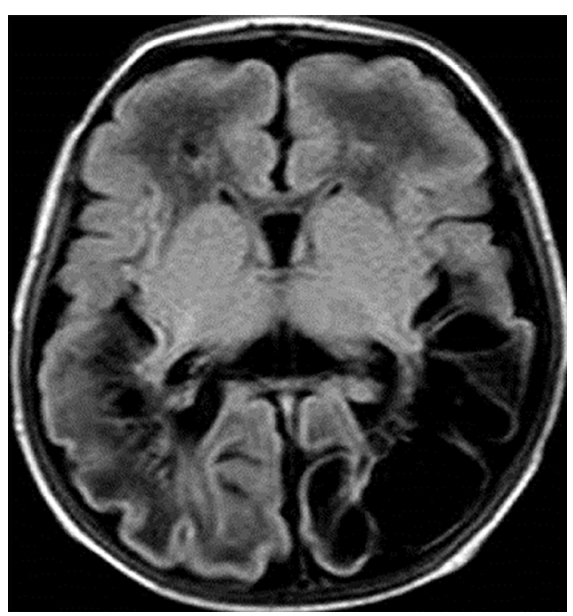

$\mathrm{H}$

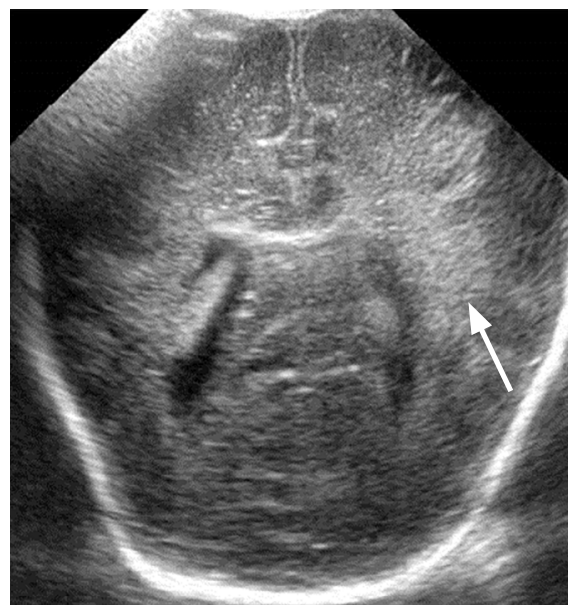

C

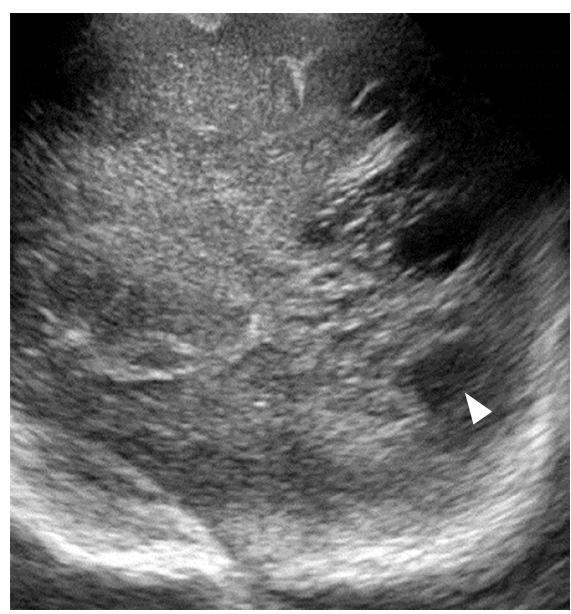

$\mathrm{F}$

Fig. 2. Preterm infant (30.9 weeks' gestational age) with macrocystic encephalomalacia after late-onset circulatory dysfunction (LCD) which occurred at 24 days of age.

A, B. At age 25 days, coronal brain sonograms show normal range of periventricular echogenicity (PVE), grade 1, next day after LCD at 24 days of age. C-F. At 35 (C, D) and $42(E, F)$ days of age, coronal and sagittal brain sonograms show an increase of PVE (C, D, arrows) and development of cystic periventricular leukomalacia and rapid progression to macrocystic encephalomalacia (E, F, arrowheads) after LCD. G, H. At 43 days of age, axial fluidattenuated inversion recovery magnetic resonance images show severe macrocystic encephalomalacia (arrows) in both parietooccipital lobes. 


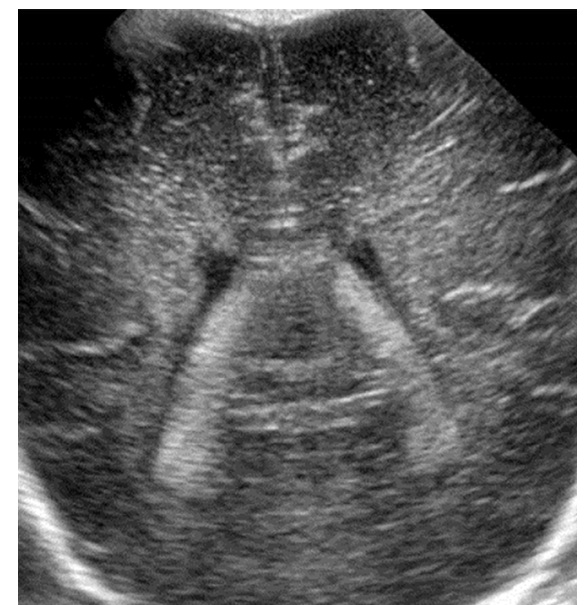

A

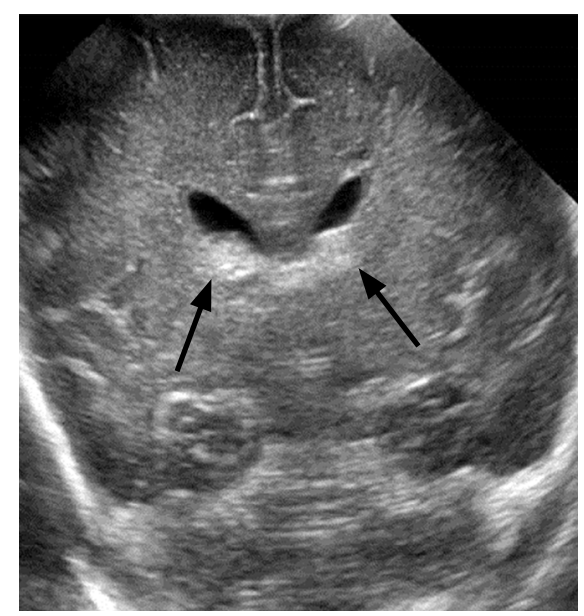

D

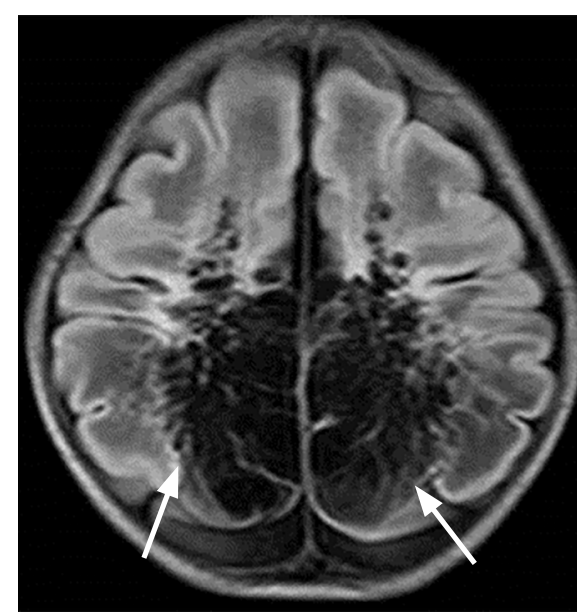

G

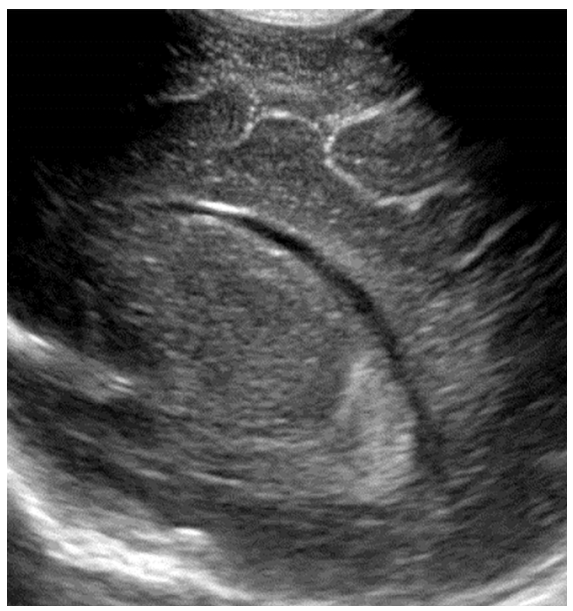

B

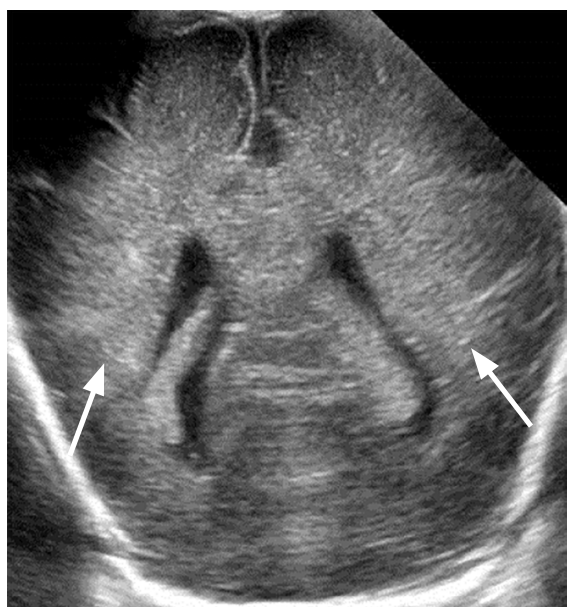

E

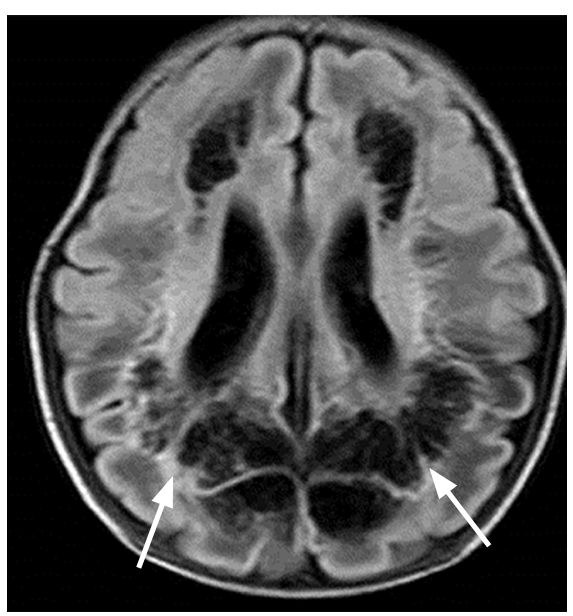

H

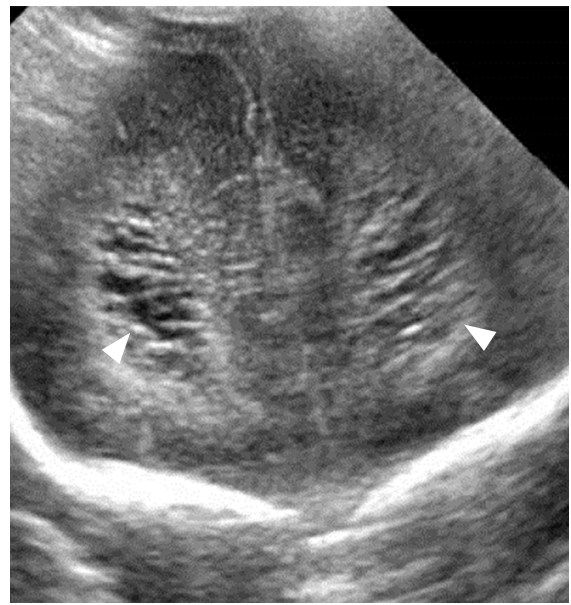

C

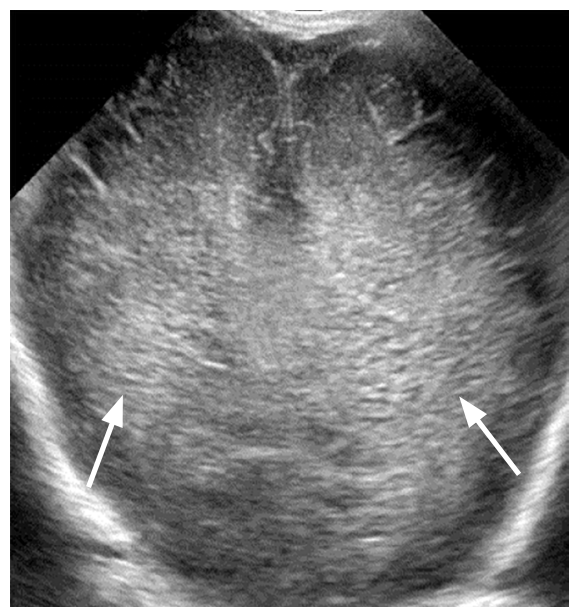

F

Fig. 3. Preterm infant (29.1 weeks' gestational age) with macrocystic encephalomalacia after late-onset circulatory dysfunction (LCD) which occurred at 31 days of age.

A, B. At 25 days of age, coronal (A) and sagittal (B) brain sonograms show a normal range of grade 1 periventricular echogenicity (PVE) before LCD. C-F. At 42 days of age, coronal brain sonograms show a marked increase of PVE (white arrows), cystic periventricular leukomalacia (arrowheads), and newly developed germinal matrix hemorrhage (black arrows) after LCD which occurred at 31 days of age. $G, H$. On day 45, axial fluid-attenuated inversion recovery magnetic resonance images show rapid progression to severe macrocystic encephalomalacia (arrows) at both of the fronto-parieto-occipital lobes. 
ORCID: Su-Mi Shin: http://orcid.org/0000-0002-7250-9573; Jee Won Chai: http:// orcid.org/0000-0003-1630-1863

\section{Conflict of Interest}

No potential conflict of interest relevant to this article was reported.

\section{Acknowledgments}

This study was supported in part by the Research Fund of the Korean Society of Ultrasound in Medicine.

\section{References}

1. Kobayashi S, Fujimoto S, Koyama N, Fukuda S, Iwaki T, Tanaka T, et al. Late-onset circulatory dysfunction of premature infants and lateonset periventricular leukomalacia. Pediatr Int 2008;50:225-231.

2. Lee JA, Choi CW, Kim EK, Kim HS, Kim BI, Choi JH. Lateonset hypotension and late circulatory collapse due to adrenal insufficiency in preterm infants with gestational age less than 32 weeks. J Korean Soc Neonatol 2011;18:211-220.

3. Kobayashi S, Fujimoto S, Fukuda S, Hattori A, Iwaki T, Koyama $N$, et al. Periventricular leukomalacia with late-onset circulatory dysfunction of premature infants: correlation with severity of magnetic resonance imaging findings and neurological outcomes. Tohoku J Exp Med 2006;210:333-339.
4. Washio Y, Uchiyama A, Nakanishi H, Totsu S, Masumoto K, Kusuda S. Hemodynamic analysis in infants with late-onset circulatory collapse. Pediatr Int 2013;55:582-588.

5. Shimokaze T, Akaba K, Saito E. Late-onset glucocorticoid-responsive circulatory collapse in preterm infants: clinical characteristics of 14 patients. Tohoku J Exp Med 2015;235:241-248.

6. Masumoto K, Kusuda S, Aoyagi H, Tamura Y, Obonai T, Yamasaki $C$, et al. Comparison of serum cortisol concentrations in preterm infants with or without late-onset circulatory collapse due to adrenal insufficiency of prematurity. Pediatr Res 2008;63:686-690.

7. Choi EJ, Sohn JA, Lee EH, Lee JY, Lee HJ, Chung HR, et al. Clinical picture of adrenal insufficiency-associated hypotension in preterm infants. J Korean Soc Neonatol 2011;18:82-88.

8. Kusuda S, Fujimura M, Sakuma I, Aotani H, Kabe K, Itani Y, et al. Morbidity and mortality of infants with very low birth weight in Japan: center variation. Pediatrics 2006;118:e1130-e1138.

9. Bassan H. Intracranial hemorrhage in the preterm infant: understanding it, preventing it. Clin Perinatol 2009;36:737-762.

10. Young RS, Hernandez MJ, Yagel SK. Selective reduction of blood flow to white matter during hypotension in newborn dogs: a possible mechanism of periventricular leukomalacia. Ann Neurol 1982; 12:445-448.

11. Ment LR, Ehrenkranz RA, Duncan CC, Lange RC. Delayed hemorrhagic infarction: a cause of late neonatal germinal matrix and intraventricular hemorrhage. Arch Neurol 1984;41:1036-1039. 